FACTA UNIVERSITATIS (NIŠ)

Ser. Math. Inform. Vol. 33, No 2 (2018), 217-230

https://doi.org/10.22190/FUMI1802217S

\title{
SOME TYPES OF $\eta$-RICCI SOLITONS ON LORENTZIAN PARA-SASAKIAN MANIFOLDS
}

\author{
Abhishek Singh and Shyam Kishor
}

\begin{abstract}
In this paper, we study some types of $\eta$-Ricci solitons on Lorentzian paraSasakian manifolds and we give an example of $\eta$-Ricci solitons on a 3-dimensional Lorentzian para-Sasakian manifold. We obtain the conditions for $\eta$-Ricci solitons on $\varphi$-conformally flat, $\varphi$-conharmonically flat and $\varphi$-projectively flat Lorentzian paraSasakian manifolds. The existence of $\eta$-Ricci solitons implies that $(M, g)$ is an $\eta$-Einstein manifold. In these cases there is no Ricci soliton on $M$ with the potential vector field $\xi$.

Keywords: $\eta$-Ricci solitons, Lorentzian para-Sasakian structure, conformal curvature, conharmonic curvature and projective curvature.
\end{abstract}

\section{Introduction}

In 1982, Hamilton [12] introduced the notion of Ricci flow to find a canonical metric on a smooth manifold. The Ricci flow is an evolution equation for metrics on a Riemannian manifold:

$$
\frac{\partial}{\partial t} g_{i j}(t)=-2 R_{i j}
$$

A Ricci soliton is a natural generalization of an Einstein metric and is defined on a Riemannian manifold $(M, g)$. A Ricci soliton is a triple $(g, V, \lambda)$ with $g$ a Riemannian metric, $V$ a vector field and $\lambda$ a real scalar such that

$$
L_{V} g+2 S+2 \lambda g=0,
$$

where $S$ is a Ricci tensor of $M$ and $L_{V}$ denotes the Lie derivative operator along the vector field $V$. The Ricci soliton is said to be shrinking, steady and expanding accordingly as $\lambda$ is negative, zero and positive, respectively. Ricci solitons have

Received July 11, 2017; accepted December 05, 2017

2010 Mathematics Subject Classification. Primary 53B30, 53C44; Secondary 53C15, 53C50. 
been studied in many contexts: on Kähler manifolds [10], on contact and Lorentzian manifolds [1, 7, 15, 21], on Sasakian [14], $\alpha$-Sasakian [15], on Kenmotsu [2] etc. In paracontact geometry, Ricci solitons firstly appeared in the paper of G. Calvaruso and D. Perrone [8]. Ricci solitons on 3-dimensional normal paracontact manifolds were studied by C. L. Bejan and M. Crasmareanu [3].

A more general notion is that of $\eta$-Ricci soliton introduced by J. T. Cho and M. Kimura [9], which was treated by C. Calin and M. Crasmareanu on Hopf hypersurfaces in complex space forms [7]. Recently, $\eta$-Ricci solitons on para-Kenmotsu manifolds were studied by A. M. Blaga [4] and $\eta$-Ricci solitons on Lorentzian paraSasakian manifolds were also studied by A. M. Blaga [5].

Let $(M, g), n=\operatorname{dim} M \geq 3$, be a connected semi-Riemannian manifold of class $C^{\infty}$ and $\nabla$ be its Levi-Civita connection. The Riemannian-Christoffel curvature tensor $R$ (see [20]), the Weyl conformal curvature tensor $C$ (see [23]), the conharmonic curvature tensor $H$ (see [16]) and the projective curvature tensor $P$ (see [23]) of $(M, g)$ are defined by

$$
\begin{aligned}
& R(X, Y) Z=\nabla_{X} \nabla_{Y} Z-\nabla_{Y} \nabla_{X} Z-\nabla_{[X, Y]} Z, \\
& C(X, Y) Z=R(X, Y) Z-\frac{1}{(n-2)}[S(Y, Z) X-S(X, Z) Y \\
& +g(Y, Z) Q X-g(X, Z) Q Y] \\
& +\frac{r}{(n-1)(n-2)}[g(Y, Z) X-g(X, Z) Y] \text {, } \\
& H(X, Y) Z=R(X, Y) Z-\frac{1}{(n-2)}[S(Y, Z) X-S(X, Z) Y \\
& +g(Y, Z) Q X-g(X, Z) Q Y], \\
& P(X, Y) Z=R(X, Y) Z-\frac{1}{(n-1)}[g(Y, Z) Q X-g(X, Z) Q Y],
\end{aligned}
$$

respectively, where $Q$ is the Ricci operator, defined by $S(X, Y)=g(Q X, Y), S$ is the Ricci tensor, $r=\operatorname{tr}(S)$ is the scalar curvature and $X, Y, Z \in \chi(M), \chi(M)$ being the Lie algebra of vector fields of $M$.

This paper is organized as follows: Section 2 consists of the basic definitions of the Lorentzian para-Sasakian manifold. In Section 3, we define Ricci and $\eta$ Ricci soliton on $(M, \varphi, \xi, \eta, g)$ and also give an example of $\eta$-Ricci solitons on a 3 -dimensional Lorentzian para-Sasakian manifold. In Section 4, we obtain the conditions for $\eta$-Ricci solitons on $\varphi$-conformally flat, $\varphi$-conharmonically flat and $\varphi$-projectively flat Lorentzian para-Sasakian manifolds. The existence of $\eta$-Ricci solitons implies that $(M, g)$ is an $\eta$-Einstein manifold. In these cases there is no Ricci soliton on $M$ with the potential vector field $\xi$. 


\section{Lorentzian para-Sasakian manifolds}

The notion of a Lorentzian para-Sasakian manifold was introduced by K. Matsumoto [17].

An $n$-dimensional differential manifold $M^{n}$ is a Lorentzian para-Sasakian $(L P$ Sasakian) manifold if it admits a $(1,1)$-tensor field $\varphi$, contravariant vector field $\xi$, a covariant vector field $\eta$ and a Lorentzian metric $g$, which satisfy

$$
\varphi^{2} X=X+\eta(X) \xi, \quad \eta(\xi)=-1,
$$

which imply

$$
\text { (a) } \varphi \xi=0, \quad(b) \quad \eta(\varphi X)=0, \quad(c) \quad \operatorname{rank}(\varphi)=n-1
$$

Then $M^{n}$ admits a Lorentzian metric $g$, such that

$$
g(\varphi X, \varphi Y)=g(X, Y)+\eta(X) \eta(Y)
$$

and $M^{n}$ is said to admit a Lorentzian almost paracontact structure $(\varphi, \xi, \eta, g)$. In this case, we have

$$
\text { (a) } g(X, \xi)=\eta(X), \quad(b) \quad \nabla_{X} \xi=\varphi X
$$

where $\nabla$ denotes the operator of covariant differentiation with respect to the Lorentzian metric $g$.

If we put

$$
\Omega(X, Y)=g(X, \varphi Y)=g(\varphi X, Y)=\Omega(Y, X),
$$

for any vector fields $X$ and $Y$, then the tensor field $\Omega(X, Y)$ is a symmetric $(0,2)$ tensor field.

Also, since the vector field is closed in an LP-Sasakian manifold, we have

$$
\left(\nabla_{X} \eta\right)(Y)=\Omega(X, Y)=g(\varphi X, Y)=\left(\nabla_{Y} \eta\right)(X), \quad \nabla_{\xi} \eta=0
$$

for any vector fields $X$ and $Y$.

Also, in an $L P$-Sasakian manifold $\left(M^{n}, \varphi, \xi, \eta, g\right)$, for any $X, Y, Z \in \chi\left(M^{n}\right)$, the following relations hold:

$$
\eta\left(\nabla_{X} \xi\right)=0, \quad \nabla_{\xi} \xi=0
$$




$$
\begin{gathered}
g(R(X, Y) Z, \xi)=\eta(R(X, Y) Z)=g(Y, Z) \eta(X)-g(X, Z) \eta(Y), \\
\eta(R(X, Y) \xi)=0, \\
R(X, Y) \xi=\eta(Y) X-\eta(X) Y, \\
L_{\xi} \varphi=0, \quad L_{\xi} \eta=0, \quad L_{\xi} g=2 g(\varphi \cdot, \cdot),
\end{gathered}
$$

where $R$ is the Riemann curvature tensor field, $L$ is the Lie derivatives and $\nabla$ is the Levi-Civita connection associated to $g$.

\section{Ricci and $\eta$-Ricci Solitons on $(M, \varphi, \xi, \eta, g)$}

Let $(M, \varphi, \xi, \eta, g)$ be paracontact metric manifolds. Consider the equation

$$
L_{\xi} g+2 S+2 \lambda g+2 \mu \eta \otimes \eta=0,
$$

where $L_{\xi}$ is the Lie derivative operator along the vector field $\xi, S$ is the Ricci curvature tensor field of the metric $g$, and $\lambda$ and $\mu$ are real constants. Writing $L_{\xi} g$ in terms of the Levi-Civita connection $\nabla$, we have:

$$
2 S(X, Y)=-g\left(\nabla_{X} \xi, Y\right)-g\left(X, \nabla_{Y} \xi\right)-2 \lambda g(X, Y)-2 \mu \eta(X) \eta(Y),
$$

for any $X, Y \in \chi(M)$, or equivalent:

$$
S(X, Y)=-g(\varphi X, Y)-\lambda g(X, Y)-\mu \eta(X) \eta(Y),
$$

for any $X, Y \in \chi(M)$. The data $(g, \xi, \lambda, \mu)$ satisfying the equation (3.1) is said to be an $\eta$-Ricci soliton on $M$ [9]; in particular, if $\mu=0,(g, \xi, \lambda)$ is a Ricci soliton [13] and it is called shrinking, steady or expanding accordingly as $\lambda$ is negative, zero or positive, respectively [11]. In [18] and [19] the the authors proved that on a Lorentzian para-Sasakian manifold $(M, \varphi, \xi, \eta, g)$, the Ricci tensor field satisfies

$$
\begin{gathered}
S(X, \xi)=(\operatorname{dim}(M)-1) \eta(X), \\
S(\varphi X, \varphi Y)=S(X, Y)+(\operatorname{dim}(M)-1) \eta(X) \eta(Y) .
\end{gathered}
$$

Again putting $X=\varphi X$ and $Y=\varphi Y$ in the equation (3.3), we get

$$
S(\varphi X, \varphi Y)=-g(X, \varphi Y)-\lambda g(\varphi X, \varphi Y),
$$

for any $X, Y \in \chi(M)$. From (3.3) and (3.4), we obtain

$$
\mu-\lambda=n-1 .
$$


Putting $X=Y=e_{i}$ in (3.3) and summing over $i=1,2, \ldots, n$, we have

$$
r=\sum_{i=1}^{n} S\left(e_{i}, e_{i}\right)=-\psi-\lambda n-\mu,
$$

where $\psi=\operatorname{tr} \varphi$.

Example 3.1. We consider the 3-dimensional manifold $M=\left\{(x, y, z) \in \mathbb{R}^{3}: z>0\right\}$, where $(x, y, z)$ are standard coordinates in $\mathbb{R}^{3}$. Let $\left\{E_{1}, E_{2}, E_{3}\right\}$ be a linearly independent frame field on $M$ given by [22]

$$
E_{1}=e^{z} \frac{\partial}{\partial x}, \quad E_{2}=e^{z-a x} \frac{\partial}{\partial y}, \quad E_{3}=\frac{\partial}{\partial z},
$$

where $a$ is a non-zero constant such that $a \neq 1$. Let $g$ be the Lorentzian metric defined by

$$
\begin{gathered}
g\left(E_{1}, E_{3}\right)=g\left(E_{2}, E_{3}\right)=g\left(E_{1}, E_{2}\right)=0, \\
g\left(E_{1}, E_{1}\right)=g\left(E_{2}, E_{2}\right)=1, \quad g\left(E_{3}, E_{3}\right)=-1 .
\end{gathered}
$$

Let $\eta$ be the 1 -form defined by $\eta(U)=g\left(U, E_{3}\right)$, for any $U \in \chi(M)$ and $\varphi$ be the $(1,1)$-tensor field defined by

$$
\varphi E_{1}=-E_{1}, \varphi E_{2}=-E_{2} \text { and } \varphi E_{3}=0 .
$$

Then, using the linearity of $\varphi$ and $g$, we have $\eta\left(E_{3}\right)=-1, \varphi^{2} U=U+\eta(U) E_{3}$ and $g(\varphi U, \varphi W)=g(U, W)+\eta(U) \eta(W)$, for any $U, W \in \chi(M)$. Thus for $E_{3}=\xi,(\varphi, \xi, \eta, g)$ defines a Lorentzian paracontact structure on $M$.

Let $\nabla$ be the Levi-Civita connection with respect to the Lorentzian metric $g$. Then we have

$$
\left[E_{1}, E_{2}\right]=-a e^{z} E_{2}, \quad\left[E_{1}, E_{3}\right]=-E_{1}, \quad\left[E_{2}, E_{3}\right]=-E_{2} .
$$

The Riemannian connection $\nabla$ of the Lorentzian metric $g$ is given by

$$
\begin{aligned}
2 g\left(\nabla_{X} Y, Z\right)= & X g(Y, Z)+Y g(Z, X)-Z g(X, Y)-g(X,[Y, Z]) \\
& -g(Y,[X, Z])+g(Z,[X, Y]),
\end{aligned}
$$

which is known as Koszul's formula. Using Koszul's formula, we can easily calculate

$$
\begin{gathered}
\nabla_{E_{1}} E_{1}=-E_{3}, \quad \nabla_{E_{1}} E_{2}=0, \quad \nabla_{E_{1}} E_{3}=-E_{1} \\
\nabla_{E_{2}} E_{1}=a e^{z} E_{2}, \quad \nabla_{E_{2}} E_{2}=-a e^{z} E_{1}-E_{3}, \quad \nabla_{E_{2}} E_{3}=-E_{2}, \\
\nabla_{E_{3}} E_{1}=0, \quad \nabla_{E_{3}} E_{2}=0, \quad \nabla_{E_{3}} E_{3}=0 .
\end{gathered}
$$

It can be easily seen that for $E_{3}=\xi,(\varphi, \xi, \eta, g)$ is a Lorentzian para-Sasakian structure on $M$. Consequently, $(M, \varphi, \xi, \eta, g)$ is a Lorentzian para-Sasakian manifold. 
Also, the Riemannian curvature tensor $R$ is given by

$$
\begin{gathered}
R(X, Y) Z=\nabla_{X} \nabla_{Y} Z-\nabla_{Y} \nabla_{X} Z-\nabla_{[X, Y]} Z, \\
R\left(E_{1}, E_{2}\right) E_{2}=\left(1-a^{2} e^{2 z}\right) E_{1}, R\left(E_{1}, E_{3}\right) E_{3}=-E_{1}, R\left(E_{2}, E_{1}\right) E_{1}=\left(1-a^{2} e^{2 z}\right) E_{2}, \\
R\left(E_{2}, E_{3}\right) E_{3}=-E_{2}, R\left(E_{3}, E_{1}\right) E_{1}=E_{3}, R\left(E_{3}, E_{2}\right) E_{2}=E_{3}+a e^{z} E_{1} .
\end{gathered}
$$

Then, the Ricci tensor $S$ is given by

$$
S\left(E_{1}, E_{1}\right)=S\left(E_{2}, E_{2}\right)=-a^{2} e^{2 z}, \quad S\left(E_{3}, E_{3}\right)=-2 .
$$

From (3.3), we obtain $S\left(E_{1}, E_{1}\right)=1-\lambda$ and $S\left(E_{3}, E_{3}\right)=\lambda-\mu$, therefore $\lambda=1+a^{2} e^{2 z}$, and $\mu=3+a^{2} e^{2 z}$. The data $(g, \xi, \lambda, \mu)$ for $\lambda=1+a^{2} e^{2 z}$, and $\mu=3+a^{2} e^{2 z}$ defines an $\eta$-Ricci soliton on the Lorentzian para-Sasakian manifold $M$.

\section{Main results}

In this section, we consider an $\eta$-Ricci soliton on $\varphi$-conformally flat, $\varphi$-conharmonically flat and $\varphi$-projectively flat Lorentzian para-Sasakian manifolds.

Let $C$ be the Weyl conformal curvature tensor of $M^{n}$. Since at each point $p \in$ $M^{n}$ the tangent space $T_{p}\left(M^{n}\right)$ can be decomposed into the direct sum $T_{p}\left(M^{n}\right)=$ $\varphi\left(T_{p}\left(M^{n}\right)\right) \oplus L\left(\xi_{p}\right)$, where $L\left(\xi_{p}\right)$ is a 1-dimensional linear subspace of $T_{p}\left(M^{n}\right)$ generated by $\xi_{p}$, we have

$$
C: T_{p}\left(M^{n}\right) \times T_{p}\left(M^{n}\right) \times T_{p}\left(M^{n}\right) \rightarrow \varphi\left(T_{p}\left(M^{n}\right)\right) \oplus L\left(\xi_{p}\right) .
$$

Let us consider the following particular cases:

(1) $C: T_{p}\left(M^{n}\right) \times T_{p}\left(M^{n}\right) \times T_{p}\left(M^{n}\right) \rightarrow L\left(\xi_{p}\right)$, i.e., the projection of the image of $C$ in $\varphi\left(T_{p}\left(M^{n}\right)\right)$ is zero.

(2) $C: T_{p}\left(M^{n}\right) \times T_{p}\left(M^{n}\right) \times T_{p}\left(M^{n}\right) \rightarrow \varphi\left(T_{p}\left(M^{n}\right)\right)$, i.e., the projection of the image of $C$ in $L\left(\xi_{p}\right)$ is zero.

$$
C(X, Y) \xi=0
$$

(3) $C: \varphi\left(T_{p}\left(M^{n}\right)\right) \times \varphi\left(T_{p}\left(M^{n}\right)\right) \times \varphi\left(T_{p}\left(M^{n}\right)\right) \rightarrow L\left(\xi_{p}\right)$, i.e., when $C$ is restricted to $\varphi\left(T_{p}\left(M^{n}\right)\right) \times \varphi\left(T_{p}\left(M^{n}\right)\right) \times \varphi\left(T_{p}\left(M^{n}\right)\right)$, the projection of the image of $C$ in $\varphi\left(T_{p}\left(M^{n}\right)\right)$ is zero. This condition is equivalent to

$$
\varphi^{2} C(\varphi X, \varphi Y) \varphi Z=0
$$

$(\operatorname{see}[6])$. 
Here the cases (1), (2) and (3) are conformally symmetric, $\xi$-conformally flat and $\varphi$-conformally flat, respectively. The cases (1) and (2) were considered in [24] and [25], respectively. The case (3) was considered in [6] for $M$ a $K$-contact manifold.

Now we will study the condition (4.2) for $\eta$-Ricci solitons on Lorentzian paraSasakian manifolds.

Definition 4.1. A differentiable manifold $\left(M^{n}, g\right), n>3$, satisfying the condition (4.2) is called $\varphi$-conformally flat.

Suppose that $\left(M^{n}, g\right), n>3$, is a $\varphi$-conformally flat Lorentzian para-Sasakian manifold. It is easy to see that $\varphi^{2} C(\varphi X, \varphi Y) \varphi Z=0$ holds if and only if

$$
g(C(\varphi X, \varphi Y) \varphi Z, \varphi W)=0,
$$

for any $X, Y, Z, W \in \chi\left(M^{n}\right)$. So by the use of (1.2), $\varphi$-conformally flat means

$$
\begin{aligned}
g(R(\varphi X, \varphi Y) \varphi Z, \varphi W)= & \frac{1}{n-2}[g(\varphi Y, \varphi Z) S(\varphi X, \varphi W) \\
& -g(\varphi X, \varphi Z) S(\varphi Y, \varphi W)+g(\varphi X, \varphi W) S(\varphi Y, \varphi Z) \\
& -g(\varphi Y, \varphi W) S(\varphi X, \varphi Z)]-\frac{r}{(n-1)(n-2)} \\
& {[g(\varphi Y, \varphi Z) g(\varphi X, \varphi W)-g(\varphi X, \varphi Z) g(\varphi Y, \varphi W)] . }
\end{aligned}
$$

Let $\left\{e_{1}, e_{2}, \ldots, e_{n-1}, \xi\right\}$ be a local orthonormal basis of vector fields in $M^{n}$; then $\left\{\varphi e_{1}, \varphi e_{2}, \ldots, \varphi e_{n-1}, \xi\right\}$ is also a local orthonormal basis. Putting $X=W=e_{i}$ in (4.3) and summing over $i=1, \ldots ., n-1$, we get

$$
\begin{aligned}
\sum_{i=1}^{n-1} g\left(R\left(\varphi e_{i}, \varphi Y\right) \varphi Z, \varphi e_{i}\right)= & \frac{1}{n-2} \sum_{i=1}^{n-1}\left[g(\varphi Y, \varphi Z) S\left(\varphi e_{i}, \varphi e_{i}\right)\right. \\
& -g\left(\varphi e_{i}, \varphi Z\right) S\left(\varphi Y, \varphi e_{i}\right)+g\left(\varphi e_{i}, \varphi e_{i}\right) S(\varphi Y, \varphi Z) \\
& \left.-g\left(\varphi Y, \varphi e_{i}\right) S\left(\varphi e_{i}, \varphi Z\right)\right]-\frac{r}{(n-1)(n-2)} \\
& \sum_{i=1}^{n-1}\left[g(\varphi Y, \varphi Z) g\left(\varphi e_{i}, \varphi e_{i}\right)-g\left(\varphi e_{i}, \varphi Z\right) g\left(\varphi Y, \varphi e_{i}\right)\right] .
\end{aligned}
$$

It can be easy to verify that

$$
\begin{gathered}
\sum_{i=1}^{n-1} g\left(R\left(\varphi e_{i}, \varphi Y\right) \varphi Z, \varphi e_{i}\right)=S(\varphi Y, \varphi Z)+g(\varphi Y, \varphi Z) \\
\sum_{i=1}^{n-1} S\left(\varphi e_{i}, \varphi e_{i}\right)=r+n-1,
\end{gathered}
$$




$$
\begin{gathered}
\sum_{i=1}^{n-1} g\left(\varphi e_{i}, \varphi Z\right) S\left(\varphi Y, \varphi e_{i}\right)=S(\varphi Y, \varphi Z), \\
\sum_{i=1}^{n-1} g\left(\varphi e_{i}, \varphi e_{i}\right)=n+1,
\end{gathered}
$$

and

$$
\sum_{i=1}^{n-1} g\left(\varphi e_{i}, \varphi Z\right) g\left(\varphi Y, \varphi e_{i}\right)=g(\varphi Y, \varphi Z) .
$$

So applying (4.5) - (4.9) into (4.4), we obtain

$$
S(\varphi Y, \varphi Z)=\left(\frac{r}{n-1}-1\right) g(\varphi Y, \varphi Z) .
$$

Using (3.6) and (3.8) in (4.10), we get

$$
(n-1) g(Y, \varphi Z)=(\psi+\mu+\lambda+n-1) g(\varphi Y, \varphi Z),
$$

for any $Y, Z \in \chi\left(M^{n}\right)$ and for $Y \mapsto \varphi Y$, we get

$$
(n-1) g(\varphi Y, \varphi Z)=(\psi+\mu+\lambda+n-1) g(Y, \varphi Z) .
$$

Adding the previous two equations, we have

$$
(\psi+\mu+\lambda+2 n-2)[g(Y, \varphi Z)-g(\varphi Y, \varphi Z)]=0,
$$

for any $Y, Z \in \chi\left(M^{n}\right)$ and follows

$$
\psi+\mu+\lambda+2 n-2=0 .
$$

Now using (3.7) in (4.24), we get

$$
\lambda=\frac{3-\psi-3 n}{2} \text { and } \quad \mu=\frac{1-\psi-n}{2} .
$$

Hence, we can state the following:

Theorem 4.1. If $(\varphi, \xi, \eta, g)$ is a Lorentzian para-Sasakian structure on the $n$ dimensional manifold $M^{n},(g, \xi, \lambda, \mu)$ is an $\eta$-Ricci soliton on $M^{n}$ and $M^{n}$ is $\varphi$ conformally flat, then

$$
\lambda=\frac{3-\psi-3 n}{2} \quad \text { and } \quad \mu=\frac{1-\psi-n}{2} .
$$


Corollary 4.1. If $(\varphi, \xi, \eta, g)$ is a $\varphi$-conformally flat Lorentzian para-Sasakian structure on the $n$-dimensional manifold $M^{n}$, then there is no Ricci soliton with a potential vector field $\xi$.

From (3.3), (3.7) and (4.11), we obtain

$$
\begin{aligned}
S(X, Y)= & -\left(\frac{\psi+n \lambda+\mu+n-1}{n-1}\right) g(X, Y) \\
& -\left(\frac{\psi+\lambda+\mu n+n-1}{n-1}\right) \eta(X) \eta(Y) .
\end{aligned}
$$

Hence, we can state the following:

Proposition 4.1. If $(\varphi, \xi, \eta, g)$ is a Lorentzian para-Sasakian structure on the $n$ dimensional manifold $M^{n},(g, \xi, \lambda, \mu)$ is an $\eta$-Ricci soliton on $M^{n}$ and $M^{n}$ is $\varphi$ conformally flat, then $\left(M^{n}, g\right)$ is an $\eta$-Einstein manifold.

Let $H$ be the conharmonic curvature tensor of $M^{n}$.

Definition 4.2. A differentiable manifold $\left(M^{n}, g\right), n>3$, satisfying the condition

$$
\varphi^{2} H(\varphi X, \varphi Y) \varphi Z=0
$$

is called $\varphi$-conharmonically flat.

Now our aim is to find the characterization of $\eta$-Ricci solitons on Lorentzian para-Sasakian manifolds satisfying the above condition.

Assume that $\left(M^{n}, g\right), n>3$, is a $\varphi$-conharmonically flat Lorentzian para-Sasakian manifold. It can be easily seen that $\varphi^{2} H(\varphi X, \varphi Y) \varphi Z=0$ holds if and only if

$$
g(H(\varphi X, \varphi Y) \varphi Z, \varphi W)=0,
$$

for any $X, Y, Z, W \in \chi\left(M^{n}\right)$. Using (1.3), $\varphi$-conharmonically flat means

$$
\begin{aligned}
g(R(\varphi X, \varphi Y) \varphi Z, \varphi W)= & \frac{1}{n-2}[g(\varphi Y, \varphi Z) S(\varphi X, \varphi W) \\
& -g(\varphi X, \varphi Z) S(\varphi Y, \varphi W)+g(\varphi X, \varphi W) S(\varphi Y, \varphi Z) \\
& -g(\varphi Y, \varphi W) S(\varphi X, \varphi Z)] .
\end{aligned}
$$

In a manner similar to the method in the proof of Theorem (4.1), choosing $\left\{e_{1}, e_{2}, \ldots, e_{n-1}, \xi\right\}$ the local orthonormal basis of vector fields in $M^{n}$, then $\left\{\varphi e_{1}, \varphi e_{2}, \ldots, \varphi e_{n-1}, \xi\right\}$ is also a local orthonormal basis. Putting $X=W=e_{i}$ in (4.17) and summing over $i=1, \ldots ., n-1$, we get

$$
\begin{aligned}
\sum_{i=1}^{n-1} g\left(R\left(\varphi e_{i}, \varphi Y\right) \varphi Z, \varphi e_{i}\right)= & \frac{1}{n-2} \sum_{i=1}^{n-1}\left[g(\varphi Y, \varphi Z) S\left(\varphi e_{i}, \varphi e_{i}\right)\right. \\
& -g\left(\varphi e_{i}, \varphi Z\right) S\left(\varphi Y, \varphi e_{i}\right)+g\left(\varphi e_{i}, \varphi e_{i}\right) S(\varphi Y, \varphi Z) \\
& \left.-g\left(\varphi Y, \varphi e_{i}\right) S\left(\varphi e_{i}, \varphi Z\right)\right] .
\end{aligned}
$$


So applying (4.5) - (4.9) into (4.18), we get

$$
S(\varphi Y, \varphi Z)=-(r+1) g(\varphi Y, \varphi Z) .
$$

Using (3.6) and (3.8) in the above equation, we get

$$
g(Y, \varphi Z)=(-\psi-\lambda n-\lambda-\mu+1) g(\varphi Y, \varphi Z),
$$

for any $Y, Z \in \chi\left(M^{n}\right)$ and for $Y \mapsto \varphi Y$, we get

$$
g(\varphi Y, \varphi Z)=(-\psi-\lambda n-\lambda-\mu+1) g(Y, \varphi Z) .
$$

Adding the previous two equations, we have

$$
(-\psi-\lambda n-\lambda-\mu+2)[g(Y, \varphi Z)-g(\varphi Y, \varphi Z)]=0,
$$

for any $Y, Z \in \chi\left(M^{n}\right)$ and follows

$$
[\psi+\lambda(n+1)+\mu-2]=0 .
$$

In view of (3.7) and (4.23), we obtain

$$
\lambda=\frac{-(\psi+n-3)}{(n+2)} \quad \text { and } \quad \mu=\frac{-\psi+n^{2}+1}{(n+2)} .
$$

Hence, we can state the following:

Theorem 4.2. If $(\varphi, \xi, \eta, g)$ is a Lorentzian para-Sasakian structure on the $n$ dimensional manifold $M^{n},(g, \xi, \lambda, \mu)$ is an $\eta$-Ricci soliton on $M^{n}$ and $M^{n}$ is $\varphi$ conharmonically flat, then

$$
\lambda=\frac{-(\psi+n-3)}{(n+2)} \quad \text { and } \quad \mu=\frac{-\psi+n^{2}+1}{(n+2)} .
$$

Corollary 4.2. If $(\varphi, \xi, \eta, g)$ is a $\varphi$-conharmonically flat Lorentzian para-Sasakian structure on the n-dimensional manifold $M^{n}$, then there is no Ricci soliton with the potential vector field $\xi$.

From (3.3), (3.7) and (4.20), we obtain

$$
\begin{aligned}
S(X, Y)= & (\psi+n \lambda+\mu-1) g(X, Y) \\
& +(\psi+n \mu+\lambda-1) \eta(X) \eta(Y) .
\end{aligned}
$$

Hence, we can state the following: 
Proposition 4.2. If $(\varphi, \xi, \eta, g)$ is a Lorentzian para-Sasakian structure on the $n$ dimensional manifold $M^{n},(g, \xi, \lambda, \mu)$ is an $\eta$-Ricci soliton on $M^{n}$ and $M^{n}$ is $\varphi$ conharmonically flat, then $\left(M^{n}, g\right)$ is $\eta$-Einstein manifold.

Let $P$ be the projective curvature tensor of $M^{n}$.

Definition 4.3. A differentiable manifold $\left(M^{n}, g\right), n>3$, satisfying the condition

$$
\varphi^{2} P(\varphi X, \varphi Y) \varphi Z=0,
$$

is called $\varphi$-projectively flat.

Assume that $\left(M^{n}, g\right), n>3$, is a $\varphi$-projectively flat Lorentzian para-Sasakian manifold. It can be easily seen that $\varphi^{2} P(\varphi X, \varphi Y) \varphi Z=0$ holds if and only if

$$
g(P(\varphi X, \varphi Y) \varphi Z, \varphi W)=0,
$$

for any $X, Y, Z, W \in \chi\left(M^{n}\right)$. Using (1.4), $\varphi$-projectively flat means

$$
\begin{aligned}
g(R(\varphi X, \varphi Y) \varphi Z, \varphi W)= & \frac{1}{n-1}[g(\varphi Y, \varphi Z) S(\varphi X, \varphi W) \\
& -g(\varphi X, \varphi Z) S(\varphi Y, \varphi W) .
\end{aligned}
$$

Similar to the proof of Theorem (4.1), we can suppose that $\left\{e_{1}, e_{2}, \ldots, e_{n-1}, \xi\right\}$ is a local orthonormal basis of vector fields in $M^{n}$, then $\left\{\varphi e_{1}, \varphi e_{2}, \ldots ., \varphi e_{n-1}, \xi\right\}$ is also a local orthonormal basis. Putting $X=W=e_{i}$ in (4.26) and summing over $i=1, \ldots . ., n-1$, we get

$$
\begin{aligned}
\sum_{i=1}^{n-1} g\left(R\left(\varphi e_{i}, \varphi Y\right) \varphi Z, \varphi e_{i}\right)= & \frac{1}{n-1} \sum_{i=1}^{n-1}\left[g(\varphi Y, \varphi Z) S\left(\varphi e_{i}, \varphi e_{i}\right)\right. \\
& \left.-g\left(\varphi e_{i}, \varphi Z\right) S\left(\varphi Y, \varphi e_{i}\right)\right] .
\end{aligned}
$$

So applying (4.5) - (4.9) into (4.27), we get

$$
n S(\varphi Y, \varphi Z)=r g(\varphi Y, \varphi Z),
$$

In view of (3.6), (3.8) and (4.28), we obtain

$$
n g(Y, \varphi Z)=(\psi+\mu) g(\varphi Y, \varphi Z),
$$

for any $Y, Z \in \chi\left(M^{n}\right)$ and for $Y \mapsto \varphi Y$, we get

$$
n g(\varphi Y, \varphi Z)=(\psi+\mu) g(Y, \varphi Z) .
$$

Adding the previous two equations, we have 


$$
(\psi+\mu+n)[g(Y, \varphi Z)-g(\varphi Y, \varphi Z)]=0,
$$

for any $Y, Z \in \chi\left(M^{n}\right)$ and follows

$$
\psi+\mu+n=0 .
$$

In view of (3.7) and (4.32), we obtain

$$
\lambda=-\psi-2 n+1 \text { and } \mu=-(\psi+n) .
$$

Hence, we can state the following:

Theorem 4.3. If $(\varphi, \xi, \eta, g)$ is a Lorentzian para-Sasakian structure on the $n$ dimensional manifold $M^{n},(g, \xi, \lambda, \mu)$ is an $\eta$-Ricci soliton on $M^{n}$ and $M^{n}$ is $\varphi$ projectively flat, then

$$
\lambda=-\psi-2 n+1 \text { and } \mu=-(\psi+n) .
$$

Corollary 4.3. If $(\varphi, \xi, \eta, g)$ is a $\varphi$-projectively flat Lorentzian para-Sasakian structure on the n-dimensional manifold $M^{n}$, then there is no Ricci soliton with the potential vector field $\xi$.

From (3.3), (3.7) and (4.29), we obtain

$$
\begin{aligned}
S(X, Y)= & \left(\frac{\psi+\mu-n \lambda}{n}\right) g(X, Y) \\
& +\left(\frac{\psi+\mu-\mu n}{n}\right) \eta(X) \eta(Y) .
\end{aligned}
$$

Hence, we can state the following:

Proposition 4.3. If $(\varphi, \xi, \eta, g)$ is a Lorentzian para-Sasakian structure on the $n$ dimensional manifold $M^{n},(g, \xi, \lambda, \mu)$ is an $\eta$-Ricci soliton on $M^{n}$ and $M^{n}$ is $\varphi$ projectively flat, then $\left(M^{n}, g\right)$ is an $\eta$-Einstein manifold.

\section{REF EREN CES}

1. C. S. Bagewadi and G. Ingalahalli: Ricci solitons in Lorentzian $\alpha$-Sasakian manifolds. Acta Math. Academiae Paedagogicae Nyíregyháziensis, 28 (2012), no. $1,59-68$.

2. C. S. Bagewadi, G. Ingalahalli and S. R. Ashoka: A Study on Ricci Solitons in Kenmotsu Manifolds. ISRN Geometry, vol. 2013, Article ID 412593, (2013), 16 . 
3. C. L. Bejan and M. Crasmareanu: Second order parallel tensors and Ricci solitons in 3-dimensional normal paracontact geometry. Anal. Global Anal. Geom., DOI:10.1007/s10455-014-9414-4.

4. A. M. Blaga: $\eta$-Ricci Solitons on Para-Kenmotsu Manifolds. Balkan J. Geom. Appl. 20 (2015), no. 1,1-13.

5. A. M. BlaGA: $\eta$-Ricci Solitons on Lorentzian Para-Sasakian Manifolds. Filomat 30 (2016), no. 2, 489-496.

6. J. L. Cabrerizo, L. M. Fernández, M. Fernández and G. Zhen: The structure of a class of $K$-contact manifolds. Acta Math. Hungar, 82 (1999), no. 4, 331-340.

7. C. Calin and M. Crasmareanu: $\eta$-Ricci solitons on Hopf hypersurfaces in complex space forms. Revue Roumaine de Mathematiques pures et appliques $\mathbf{5 7}$ (2012), no. 1, 55-63.

8. G. Calvaruso and D. Perrone: Geometry of H-paracontact metric manifolds. arxiv:1307.7662v1.2013.

9. J. T. Сhо and M. Kimura: Ricci solitons and real hypersurfaces in a complex space form. Tohoku Math. J. 61 (2009), no. 2, 205-212.

10. O. Chodosh and F. T. -H. Fong: Rotational symmetry of conical Kähler-Ricci solitons. arxiv:1304.0277v2.2013.

11. B. Chow, P. Lu and L. Ni: Hamilton's Ricci Flow. Graduate Studies in Mathematics, AMS, Providence, RI, USA 77 (2006).

12. R. S. Hamilton: Three-manifolds with positive Ricci curvature. J. Diff. Geom., 17 (1982), no. 2, 255-306.

13. R. S. Hamilton: The Ricci flow on surfaces. Mathematics and General Relativity (Santa Cruz, CA, 1986), Contemp. Math. 71, American Math. Soc. 1988, 237-262.

14. C. He and M. ZHU: The Ricci solitons on Sasakian manifolds. arxiv1109.4407v2.2011.

15. G. Ingalahalli and C. S. Bagewadi: Ricci solitons in $\alpha$-Sasakian manifolds. ISRN Geometry, vol. 2012, Article ID 421384, (2012), 1-13.

16. Y. ISHII: On conharmonic transformations. Tensor N.S, 7 (1957), 73-80.

17. K. Matsumoto: On Lorentzian paracontact manifolds. Bull. of Yamagata Univ. Nat. Sci, 12 (1989), no. 2, 151-156.

18. K. Matsumoto and I. Minai: On a certain transformation in a Lorentzian paraSasakian manifold. Tensor N. S., 47 (1988), 189-197.

19. I. Minai, A. A. Shaikh and U. C. De: On Lorentzian para-Sasakian manifolds. Rendiconti del Seminario Matematico di Messina, Serie II (1999).

20. C. OzguR: $\phi$-conformally flat Lorentzian para-Sasakian manifolds. Radovi Mathematicki, 12, (2003), 99-106.

21. M. M. TRIPATHI: Ricci solitons in contact metric manifolds. http://arxiv.org/abs/0801.4222.

22. S. K. YADAV and D. L. SuthaR: Some global properties of LP-Sasakian manifolds. Fundamental Journal of Mathematics and Mathematical Sciences, 3 (2015), no. 1, 69-82.

23. K. Yano and M. Kon: Structures on manifolds. Series in Pure Mathematics, World Scientific Publishing Co., Singapore 3 (1984). 
24. G. Zhen: On conformal symmetric $K$-contact manifolds. Chinese Quart. J. of Math, 7 (1992), 5-10

25. G. Zhen, J. L. Cabrerizo, L. M. Fernández and M. Fernáandez: On $\xi$ conformally flat contact metric manifolds. Indian J. Pure Appl. Math, 28 (1997), 725-734.

Abhishek Singh

Department of Mathematics and Astronomy

University of Lucknow,

Lucknow-226007,

India.

lkoabhi27@gmail.com

Shyam Kishor

Department of Mathematics and Astronomy,

University of Lucknow,

Lucknow-226007,

India.

skishormath@gmail.com 